EPJ Web of Conferences 59, 18005 (2013)

DOI: $10.1051 /$ epjconf/20135918005

(C) Owned by the authors, published by EDP Sciences, 2013

\title{
Laser target using continuous supersonic jet in vacuum
}

\author{
Yujin Ogata ${ }^{1, a}$, Kazumasa Takahashi ${ }^{1}$, Hajime Kuwabara ${ }^{2}$, \\ Mitsuo Nakajima ${ }^{1}$ and Kazuhiko Horioka ${ }^{1}$ \\ ${ }^{1}$ Department of Energy Sciences, Tokyo Institute of Technology, \\ Nagatsuta 4259, Midori-ku, Yokohama 226-8502, Japan \\ 2 Yokohama Engineering Center, IHI Corporation, 1, Shin-nakaharacho, Isago-ku, Yokohama \\ 235-8501, Japan
}

\begin{abstract}
For an extreme ultraviolet (EUV) light source, Sn plasmas, which can produce a strong radiation with high conversion efficiency at $13.5 \mathrm{~nm}$, attract attention. The purpose of this study is to establish technology to form and recover a tin vapor supersonic jet in vacuum and to make clear the interaction between the supersonic free jet and an incident laser. In order to make the tin vapor, a tantalum crucible is inductively heated up and the radiation loss is compared with the input energy. We observe the shock waves in argon jet emitted from a supersonic nozzle by a Schlieren image. In the future, we will increase the number of shields and heat the tantalum crucible to the boiling point of tin. We are planning to make a study on the interaction process between laser and the free jet.
\end{abstract}

\section{INTRODUCTION}

As the next generation optical lithography for advanced large-scale integrated circuit, EUV light source at $13.5 \mathrm{~nm}$ is under development now. In a practical EUV lithography system, an average EUV power of $180 \mathrm{~W}$ in $2 \%$-bandwidth around $13.5 \mathrm{~nm}$ is required at the intermediate focus. EUV light sources can be roughly classified into two groups; that is based on a laser-produced plasma (LPP) and a discharge produced plasma (DPP).

Figure 1 shows a basic concept of LPP. In the concept, a high energy pulsed laser irradiates a target, which produces a high energy density plasma as the light source with a particular spectrum range.

LPP has three advantages. First, because there is not a structure interrupting the optical transport, we can get a large collecting solid angle around the plasma source. Next, a control of the source size is easy and an angle distribution of EUV power is uniform. Finally, the plasmas can be produced at optimum density, if we can control the target condition [1-4].

In EUV lithography, a Mo/Si multilayer mirror which has a sensitivity peak at $13.5 \mathrm{~nm}$ is used, in which $1 \%$-bandwidth around $13.5 \mathrm{~nm}$ is effective. We have to use an element having an emission spectrum in this domain as the target. Sn plasmas can produce a brilliant light with high in-band conversion efficiency at $13.5 \mathrm{~nm}$.

A solid, a liquid, and a gas can be the plasma medium. However, the solid target is too dense and generates a lot of debris that damage the collector optics and limit the lifetime of the optical system. Moreover, a highly repetitive supply, that is indispensable to the high average power output, is predicted to be difficult in case of the solid target.

\footnotetext{
${ }^{a}$ e-mail: takahashi.k.ax@m.titech.ac.jp
}

This is an Open Access article distributed under the terms of the Creative Commons Attribution License 2.0, which permits unrestricted use, distribution, and reproduction in any medium, provided the original work is properly cited. 


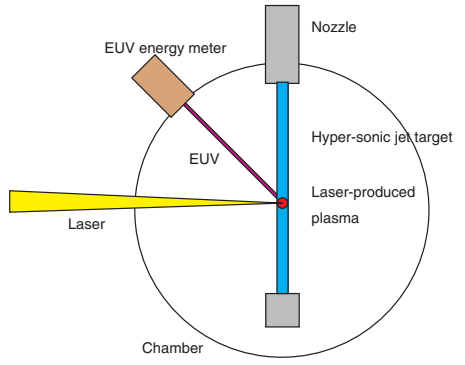

Figure 1. Basic concept of laserproduced plasma (LPP) using continuous free jet.

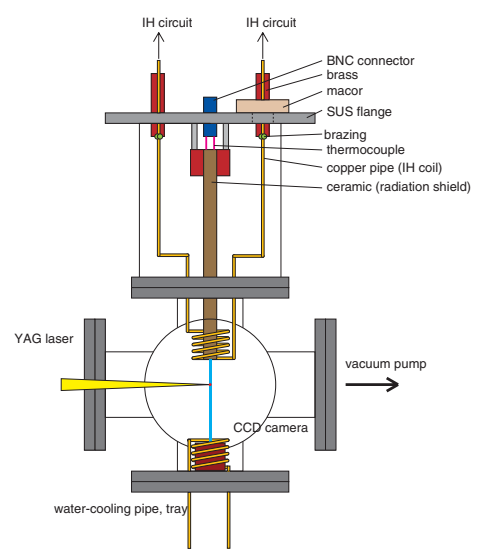

Figure 2. Schematic of experimental Figure 3. Schematic of miniature setup.

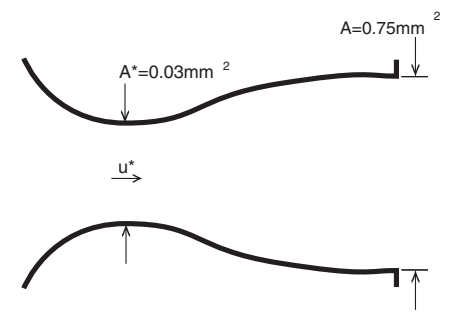

supersonic nozzle.

On the other hand, in case of a liquid (Sn) droplet target, the laser spot size is larger than the target size, so a part of the beam passes outside the droplet. In order to improve the coupling efficiency between the long-wavelength $\mathrm{CO}_{2}$ laser and the micro-target, $\mathrm{Sn}$ droplets have to be expanded by 10-20 times in advance by another laser. However, the double-pulse laser irradiation method is considered to be difficult to operate the system practically [1].

Instead of them, we propose a continuous gas jet as the laser target. By the gas target system with density region of $\sim 10^{19} / \mathrm{cm}^{3}$, we can suppress the debris almost completely and realize a high coupling efficiency between $\mathrm{CO}_{2}$ laser and the target.

The purpose of this study is to establish the technological basis to form and recover a supersonic metal vapor jet continuously in vacuum. With controlled tin vapor supersonic jet, we can suppress the debris; i.e., the formation of micro-droplets and fast neutral particles. At the same time, by controlling the density profile of the jet, we can expect to get high conversion efficiency. Also, by continuous supply and recovery of the vapor, we can realize highly reproducible and repetitive operation of the light source.

An important issue that we should make clear experimentally is the interaction between the supersonic free jet and laser. Because a high temperature domain is generated by the laser irradiation in supersonic jet, the temperature rises locally and the local Mach number of the jet decreases. Naturally a transonic and subsonic domain should grow up. Then it is expected that disturbance induced in the interaction domain reaches the upper region of the flow. Shock waves reaching the upper region of the flow should interrupt the mass and momentum recovering of the jet.

\section{EXPERIMENT}

Figure 2 shows a schematic diagram of the experimental device. The device consists of a vacuum system, a Nd:YAG laser, a CCD camera, and a measurement device for the temperature history. The experimental device has a miniature nozzle which discharges a tin vapor jet into the vacuum, a saucer which collects the jet, and an induction heater for tin vapor. The nozzle was pointed downward so that the jet does not interfere with the induction heating $(\mathrm{IH})$ coil. We made the induction heating coil with copper pipe to cool itself with a water flow. 


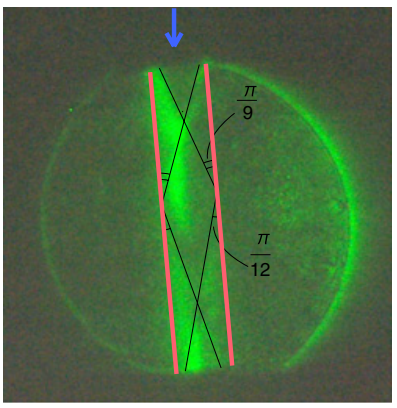

Figure 4. Schlieren photograph of argon jet.

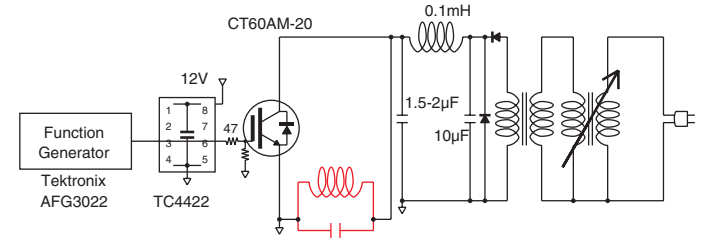

Figure 5. Circuit diagram of induction heating.

\subsection{Supersonic nozzle}

We use tantalum as the nozzle and crucible materials. Figure 3 shows the geometry of supersonic nozzle. The tin vapor is hydrodynamically accelerated with the nozzle, accompanied by condensation of the vapor.

We set the nozzle throat diameter as $0.8[\mathrm{~mm}]$ and the exit diameter as $3.0[\mathrm{~mm}]$ to realize $M=5.75$.

Figure 4 shows a Schlieren photograph of argon jet. We observed the density gradient and shock wave of argon jet by a carefully tuned Schlieren photograph.

\subsection{Induction heating}

Figure 5 shows a circuit diagram of the heating device. A high frequency electric current is provided by driving an LC resonator operated by IGBT switches. We put a capacitor in the heater coil in parallel to resonantly drive the high frequency current.

\subsection{Radiation shields}

The radiation heat flux $\dot{q}$ is estimated with

$$
\dot{q}=\frac{\sigma}{(n+1)\left(\frac{2}{\varepsilon}-1\right)}\left(T_{j}^{4}-T_{0}^{4}\right)
$$

where $\sigma$ is the Stefan-Boltzmann constant, $n$ is the number of the radiation shield, $\varepsilon$ is the emissivity.

Silica-glass fiber tapes are used for the radiation shields. Figure 6 shows the estimated heat flux of radiation. As expected, radiation is dominant in high-temperature range, because the heat flux varies with fourth power of the temperature.

Temperature history of the tantalum crucible with $n=1$ was measured as a function of input power. Figure 7 shows the temperature history of crucible with transformer voltage as a parameter. By converting a vertical axis (crucible temperature) into energy $Q=M c T$ and differentiating it in time, total input power to the crucible and the energy loss power were found at each temperature. That is shown in Figure 8. The upper part of the graph shows change of the net energy at the time of induction heating. The lower part of the graph is the net energy loss when the electric transformer was turned off. In other words, the history reflects the energy flow that is lost in radiation and heat conduction. As a result, the heat flux to and from the tantalum crucible was calculated with transformer voltage as a parameter.

Next, we compared the temperature history with an analytical equation of the heat losses. Factors are derived by fitting of this curve to the expression of heat conduction and radiation. They are expressed as 


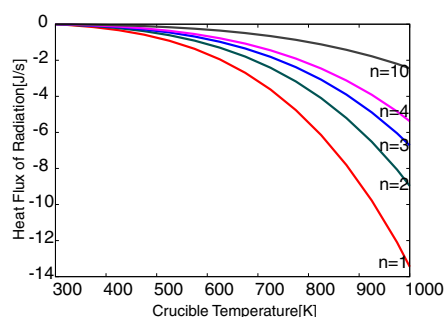

Figure 6. Theoretical value of radiation flux versus crucible temperature ( $n$ shows the number of shield).

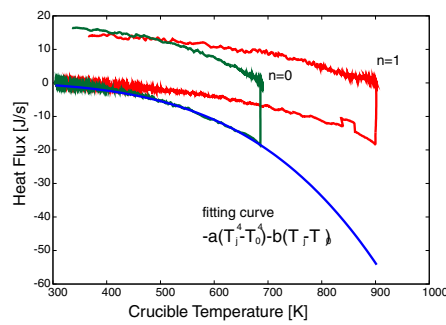

Figure 9. Comparison of heat flux for $n=0$ (and $n=1$ ). $a=$ $8.0 \times 10^{-11}, b=2.0 \times 10^{-3}$ are calculated.

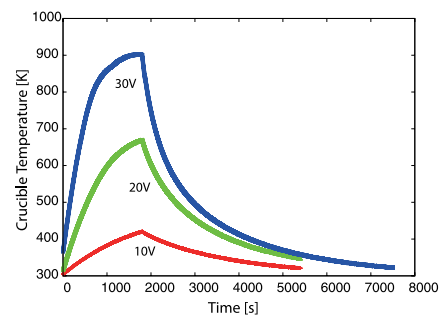

Figure 7. Temperature history tantalum crucible $(n=1)$.
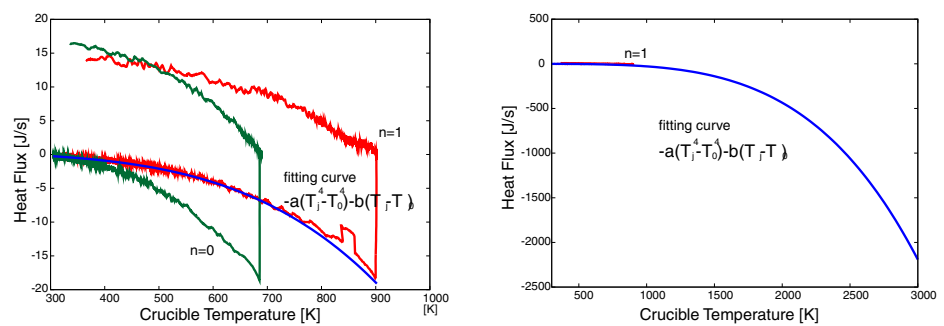

Figure 10. Radiation flux at $\varepsilon=0.52$ Figure 11. Derived heat loss of and $n=1 . a=2.7 \times 10^{-11}$ and $b=$ the device, which indicates loss is $2.0 \times 10^{-3}$ are derived by fitting the $1850[\mathrm{~W}]$ at $2875[\mathrm{~K}]$ for $n=1$. history.

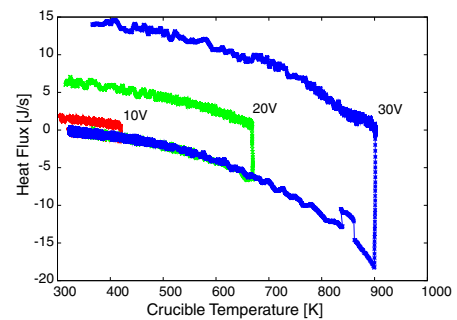

Figure 8. Heat flux of the crucible and the energy loss.

follows,

$$
\dot{q}=-a\left(T_{j}^{4}-T_{0}^{4}\right)-b\left(T_{j}-T_{0}\right)
$$

where $a$ is a factor of radiation and $b$ is a factor of heat conduction. In the case of $n=0, a=8.0 \times 10^{-11}$ and $b=2.0 \times 10^{-3}$ are calculated (Fig. 9). By fitting the temperature history for the $n=1$ case, tantalum crucible emissivity was estimated to be 0.52 .

When we put $\varepsilon=0.52$, the radiation flux can be calculated for $\mathrm{n}=1$. In this condition, $a=$ $2.7 \times 10^{-11}$ and $b=2.0 \times 10^{-3}$ are derived. The heat loss is calculated to be $1850[\mathrm{~W}]$ by extrapolating this curve to the boiling temperature $2875[\mathrm{~K}]$ of tin (as shown in Fig. 11).

\section{CONCLUDING REMARKS}

We proposed a continuous supersonic jet as the target of laser plasma interaction experiments. We observed the density gradient of argon jet emitted from the supersonic nozzle by Schlieren photograph. We made a heating system for Sn vapor jet formation in vacuum using induction heating. We compared the characteristic of the radiation loss with the input power to derive effective value of radiation loss of the tantalum crucible. The tantalum crucible emissivity was found to be 0.52 . The heat loss was calculated to be $1850[\mathrm{~W}]$ by fitting and extrapolating this curve to $2875[\mathrm{~K}]$ at $n=1$.

In the future, we will increase the number of shields and heat the tantalum crucible to the tin boiling point. Next to the jet formation, we do experiments to irradiate a laser to the tin vapor free jet and to investigate the interaction process between the laser and the supersonic free jet. 


\section{IFSA 2011}

\section{References}

[1] D. Nakamura, T. Akiyama, A. Takahashi, and T. Okada, JLMN-Journal of Laser Micro/Nanoengineering Vol.3, 196 (2008)

[2] D. Nakamura, K. Tamaru, T. Akiyama, A. Takahashi, and T. Okada, The Review of Laser Engineering Supplemental 36, 1129 (2008)

[3] P. Hayden, A. Cummings, N. Murphy, G. O’Sullivan, P. Sheridan, J. White, and P. Dunne, J. Appl. Phys. 99, 093302 (2006)

[4] T. Higashiguchi, M. Hamada, and S. Kubodera, Rev. Sci. Instrum. 78, 036106 (2007) 